\title{
BREAST IMAGES
}

\section{Double Localization and Multiple Recurrences of Dermatofibrosarcoma Protuberans of the Female Breast: A Rare Clinical Case}

\author{
Valentina Territo, MD, ${ }^{*}$ Vincenza Morello, MD ${ }^{\dagger}$ Anna Maria Caruso, MD,* \\ Giuseppe Livio Angelo, MD, ${ }^{*}$ and Gaetano Giuseppe Di Vita, MD* \\ *General Surgery Unit - Department of Surgical, Oncological and Stomatological Sciences, Università \\ di Palermo, Palermo, Italy; ‘rea funzionale di Anatomia Patologica - Dipartimento di Scienze per la \\ promozione della salute e materno - infantile “G. D' Alessandro, Università di Palermo, Palermo, \\ Italy
}

In October 2008, in another hospital, a 53-year-old woman underwent to an excision of two masses in the left breast: one of $14 \mathrm{~mm}$ in the retroareolar side and another of $8 \mathrm{~mm}$ near the skin surface of the inframammary sulcus. The histologic assay of the two neoplasms led to the diagnosis of dermatofibrosarcoma protuberans (DFSP), which arrived at $1 \mathrm{~mm}$ from the surgical resection margins. In June 2010, it was

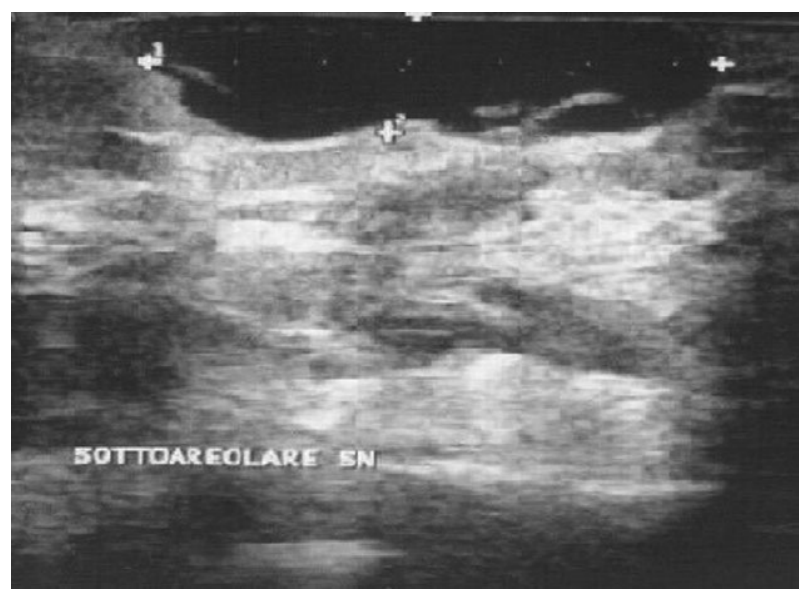

Figure 1. Ultrasonography: ovular and irregular anechoic lump of about $37 \times 10 \mathrm{~mm}$ in the left breast.

Address correspondence and reprint requests to: Gaetano Di Vita, Department of Surgical, Oncological and Stomatological Sciences, Division of General Surgery, University of Palermo, Italy, Via Liborio Giuffrè, 5, Palermo, Italy, or e-mail: divitagaetano@libero.it

DOI: $10.1111 /$ tbj.12401

C 2015 Wiley Periodicals, Inc., 1075-122X/15

The Breast Journal, Volume 21 Number 3, 2015 308-309 demonstrated the presence in the left retroareolar side of a neoplasm of $20 \mathrm{~mm}$ that was excised. The histologic assay showed a recurrent DFSP with free margins. In January 2011, the patient came to our clinic for a lump in the surgical retroareolar scar. On physical examination, in left retroareolar region, a $3 \mathrm{~cm}$ firm neoplasm with a combination of blue and red colors was present at the previous surgical scar. After an ultrasonography (Fig. 1) and a mammography (Fig. 2), we performed a mastectomy, considering anxiety of the patient, previous double localization, early recurrences in spite of the free margins, and size of the residual breast. Histologic diagnosis was of a

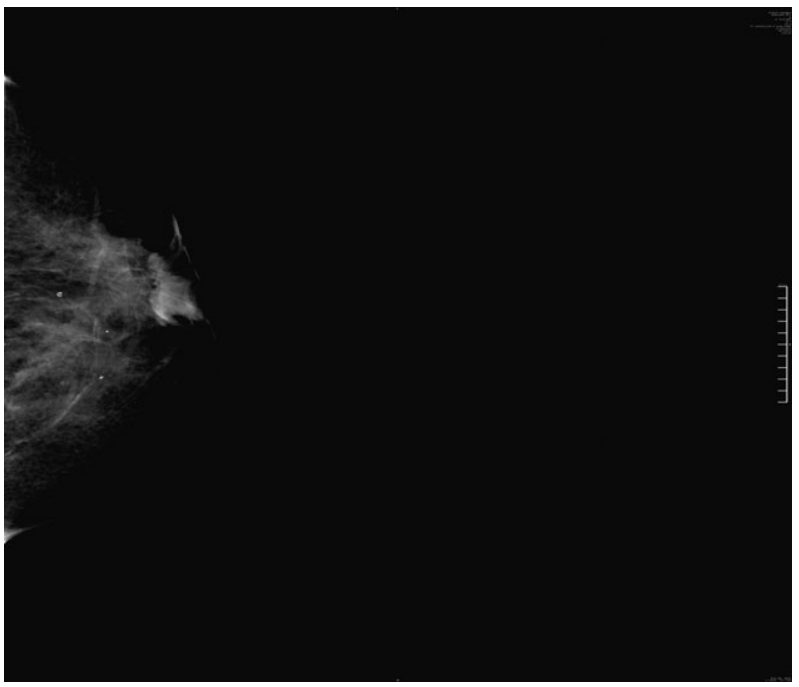

Figure 2. Mammography: ovular and irregular neoplasm of about $28 \times 16.7 \mathrm{~mm}$ in the left retroareolar region. 


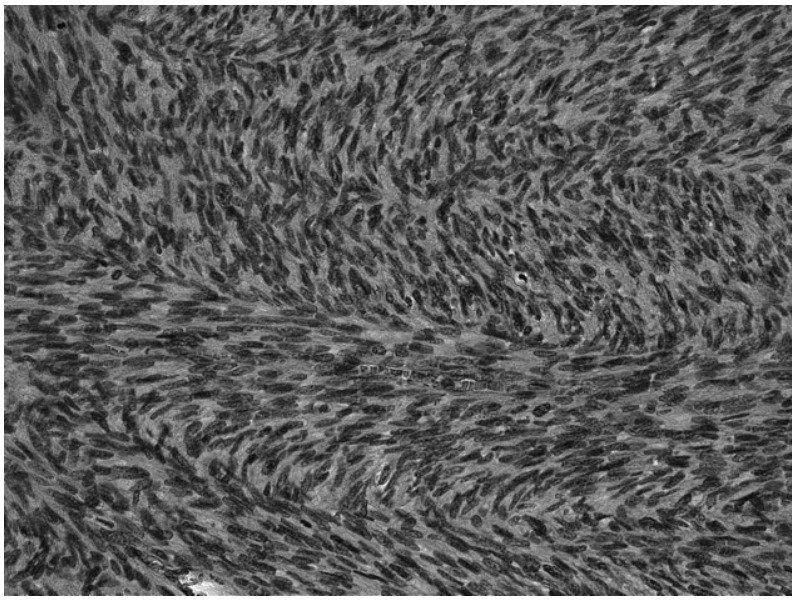

Figure 3. In a higher power field tumor fascicles, composed by uniformly monotonous spindle cells, interdigitate with collagen bundles mimicking a "low grade" fibrosarcoma-like aspect: note the low mitotic activity and the little nuclear pleomorphism.

recurrent DFSP that in some field was mimicking a "low grade" fibrosarcoma-like aspect (Fig. 3). Mitotic activity was not exceeding three mitoses/10 HPF. Immunohistochemically, tumor cells were extensively positive for CD34 (Fig. 4). No additional adjuvant treatment was done. After 24 months, the patient underwent to a breast reconstruction. Actually, our patient is well 30 months after our surgery.

The localization of DFSP in the skin of the breast (DFSP-Br) is rare. In our review of the last 10 years, only 21 cases of DFSP-Br were reported and it commonly rushes between the second to fifth decades of life. Sometimes, differentiating DFSP from other dermatofibromas using CD34 and the other antigens

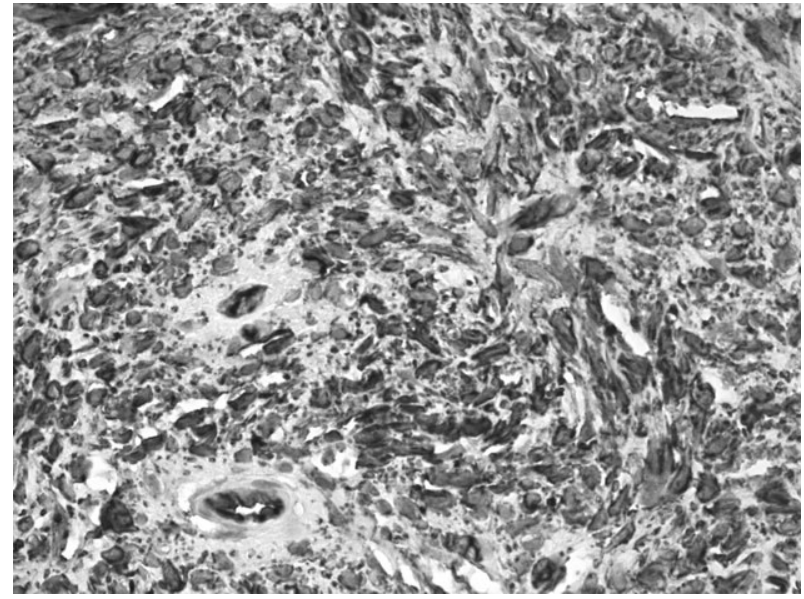

Figure 4. Intense and extensive CD34-immunostaining of tumor cells of dermatofibrosarcoma protuberans.

immunochemistry remains difficult; therefore, in these cases, a search of some genetic alterations may be useful. About the therapy, the recommended treatment of DFSP is a wide surgical excision with pathologic negative margins. Despite optimal surgical management, local recurrences are detected in $25 \%$ of patients. A problem not to be underestimated in case of DFSP-Br is the esthetic one, which can be felt particularly in the case of reoperation for recurrence, especially in small breasts. Since wide excision usually causes noteworthy distortion and leaves patient with significant cosmetic problems, reconstructive procedure is required in almost every istances. Due to the significant recurrences, even if with free pathologic margins, a long-term postoperative follow-up is mandatory.

\title{
Combined Fibroadenoma and Tubular Adenoma of the Breast: Rare Presentation that Confirms Common Histogenesis
}

\author{
Joško Bezić, MD, MSc, Ivana Karaman, MD, and Dinka Šundov, MD \\ Institute of Pathology, Forensic Medicine and Cytology, Clinical Hospital Center, Split, Croatia
}

Address correspondence and reprint requests to: Joško Bezić, Institute of Pathology, Forensic Medicine and Cytology, Clincal Hospital Center Split, Spinčićeva 1, 21000 Split, Croatia, or e-mail: jbezic@mefst.hr

DOI: $10.1111 /$ tbj.12400

(C) 2015 Wiley Periodicals, Inc., 1075-122X/15

The Breast Journal, Volume 21 Number 3, 2015 309-311
A 19-year-old woman presented with palpable, Apainless nodule of her left breast detected by selfexamination. On physical examination, a lump of $2 \mathrm{~cm}$ in diameter was palpable in the middle outer portion of the left breast. The lump was mobile with 